\title{
Erratum to: Simulation of the Three-Dimensional Hinge Flow Fields of a Bileaflet Mechanical Heart Valve Under Aortic Conditions
}

\author{
Hélène A. Simon, ${ }^{1}$ Liang Ge, ${ }^{3}$ Iman Borazjani, ${ }^{4}$ Fotis Sotiropoulos, ${ }^{4}$ and Ajit P. Yoganathan ${ }^{1,2}$ \\ ${ }^{1}$ School of Chemical and Biomolecular Engineering, Georgia Institute of Technology, Atlanta, GA, USA; ${ }^{2}$ The Wallace \\ H. Coulter Department of Biomedical Engineering, Georgia Institute of Technology, Atlanta, GA, USA; ${ }^{3}$ University of \\ California San Francisco, San Francisco, CA, USA; and ${ }^{4}$ St. Anthony Falls Laboratory, Department of Civil Engineering, \\ University of Minnesota, Minneapolis, MN, USA
}

\section{Erratum to: Annals of Biomedical Engineering}

DOI 10.1007/s10439-009-9857-0

Dr. Iman Borazjani was inadvertently omitted as an author of the publication. The author list appears correctly here.

Address correspondence to Ajit P. Yoganathan, The Wallace H. Coulter Department of Biomedical Engineering, Georgia Institute of Technology, 313 Ferst Drive, BME Building, Room 2119, Atlanta, GA 30332-0535; USA. Electronic mail: ajit.yoganathan@bme. gatech.edu

The online version of the original article can be found under doi: 10.1007/s10439-009-9857-0. 